\title{
Acknowledgment of Ad Hoc Reviewers (2018)
}

The Editor would like to thank the following persons who served as reviewers for History of Psychology, from September 2017 through September 2018.

John Andrick

Alexandra BacopoulosViau

Maite Barrios

Carla Bittel

Jeremy Blatter

Adrian C. Brock

Sebastiaan Broere

Michael Cole

Tabea Cornel

Paul Croce

Nancy L. Digdon

Rory DuPlessis

Greg Eghigian

Rogelio Escobar

Ivan Flis

Luciano Nicolás García

Fernando Gonzalez Rey

Andrea Graus
Horst U. K. Gundlach

Gary Hatfield

Vincent W. Hevern

James Hoesterey

Katherine Hubbard

Deborah Johnson

Ann Johnson

Russell D. Kosits

Robert Kugelmann

Sofie Lachapelle

Christian Laes

James T. Lamiell

Debra Lindsay

Wahbie Long

Johann Louw

Sarah Marks

Luciano Mecacci

Raul Necochea

Serge Nicolas
Matthew Oram

Elissa Rodkey

Mariano Ruperthuz

Jonathan Sadowsky

Kathryn Schoefert

Sonu Shamdasani

Lothar Spillmann

Henderikus J. Stam

Warren Thorngate

Brandi Townsend

René van der Veer

Julia Vassilieva

Tom Vinci

Richard von Mayrhauser

Sarah Walsh

Wen-Ji Wang

Anton Yasnitsky

Jacy L. Young

Ekaterina Zavershneva 